\title{
A New Kinetic Mode During the Austenite-to-Ferrite Transformation in Fe-Mn and Fe-Mn-Mo Alloys
}

\author{
Cong-Yu Zhang ${ }^{1} \cdot$ Hao Chen ${ }^{1} \cdot$ Jia-Ning Zhu ${ }^{1} \cdot$ Chi Zhang $^{1} \cdot$ Zhi-Gang Yang $^{1}$
}

Received: 26 October 2019 / Revised: 21 December 2019 / Published online: 20 March 2020

(C) The Chinese Society for Metals (CSM) and Springer-Verlag GmbH Germany, part of Springer Nature 2020

\begin{abstract}
The kinetics of isothermal austenite $(\gamma)$-to-ferrite $(\alpha)$ transformation at various temperatures in $\mathrm{Fe}-2 \mathrm{Mn}$ and $\mathrm{Fe}-2 \mathrm{Mn}-0.5 \mathrm{Mo}$ (wt\%) alloys is investigated via dilatometry experiments and phase-field modeling. It was interestingly found that Mo addition has a marginal effect on the transformation kinetics. Besides the well-known partitioning and partitionless transformation modes, a new kinetic mode, in which interface migration is controlled by interfacial diffusion, was identified. The phase-field model with considering interfacial segregation could well predict the transformation kinetics and the kinetic mode transition.
\end{abstract}

Keywords Steel $\cdot$ Austenite $\cdot$ Ferrite $\cdot$ Diffusion $\cdot$ Solute drag

\section{Introduction}

The austenite $(\gamma)$-to-ferrite $(\alpha)$ transformation is one of the most important diffusional solid-state phase transformations in steels processing. The kinetics of $\gamma \rightarrow \alpha$ transformation is in principle controlled by both element diffusion and interface migration [1,2], while in many cases it is dominated by either element diffusion or interface migration [3-5]. $\mathrm{Fe}-X$ alloys $(X$ represents substitutional elements, such as $\mathrm{Mn}, \mathrm{Ni}, \mathrm{Mo}$, etc.) are ideal model systems for investigating the mechanism of the $\gamma \rightarrow \alpha$ transformation. The kinetics of the $\gamma \rightarrow \alpha$ transformation upon continuous cooling in $\mathrm{Fe}-X$ alloys has been widely studied, and it was found there is a kinetic transition from partitioning to partitionless transformation (e.g., massive transformation). Due to the very slow diffusion of $X$ in $\gamma$, the partitioning $\gamma \rightarrow \alpha$ transformation is very sluggish. The partitionless massive transformation is fully controlled by interface migration, which has an interface velocity in the order of magnitude about $10^{-6}$ to $10^{-5}$ $\mathrm{m} / \mathrm{s}$ and generally keeps this interface velocity during the transformation $[6,7]$. Thermodynamically speaking, the

Available online at http://link.springer.com/journal/40195.

Hao Chen

hao.chen@mail.tsinghua.edu.cn

Key Laboratory of Advanced Materials of Ministry of Education, School of Materials Science and Engineering, Tsinghua University, Beijing 100084, China transition from partitioning to partitionless transformation should occur at the critical temperature $T_{0}$ where the Gibbs energy of $\alpha$ phase is equal to that of $\gamma$ phase with same concentration. However, in fact, the experimental kinetic transition temperature can deviate from $T_{0}$ due to the complex interaction between $X$ and interface migration, which was considered to be due to the solute drag effect of $X$ [8-10].

The solute drag effect of $X$, such as Mn or Mo, has been widely studied in $\mathrm{Fe}-\mathrm{C}-X$ alloys [11-13]. It is far less studied in interstitial-free $\mathrm{Fe}-X$ alloys, even though $\mathrm{C}$ was reported to have important effects on the interaction between $X$ and migrating interface $[14,15]$. Besides, currently, much effort has been made on studying the $\gamma \rightarrow \alpha$ transformation in $\mathrm{Fe}-X$ alloys during continuous cooling from single $\gamma$ phase region [7, 16, 17]. Nevertheless, the isothermal $\gamma \rightarrow \alpha$ transformation in $\mathrm{Fe}-X$ alloys is rarely investigated. It was usually accepted that the kinetic mode of $\gamma \rightarrow \alpha$ transformation could be either fast partitionless mode or the sluggish partitioning mode. However, in this study, we experimentally observed a new kinetic mode in which interface migration was controlled by interfacial diffusion. A phase-field model with considering interfacial segregation was proposed to successfully predict this new kinetic mode. 


\section{Experimental}

The kinetics of isothermal $\gamma \rightarrow \alpha$ transformation in $\mathrm{Fe}-2 \mathrm{Mn}$ and $\mathrm{Fe}-2 \mathrm{Mn}-0.5 \mathrm{Mo}$ alloys (all in wt $\%$ ), as well as the alloying effects of Mn and Mo, will be experimentally and theoretically studied. The chemical composition and $T_{0}$ of the two alloys investigated in this study are listed in Table 1.

The standard cylinder specimens with dimensions of $4 \mathrm{~mm}$ in diameter and $10 \mathrm{~mm}$ in length are prepared for dilatometry experiments. The dilation of the specimens during heat treatment is measured by a Bahr 805A-type dilatometer. The specimens were first heated up to $950{ }^{\circ} \mathrm{C}$ for full austenization at a heating rate of $5 \mathrm{~K} / \mathrm{s}$ and held for $5 \mathrm{~min}$. Then, the specimens were cooled down at a cooling rate of $20 \mathrm{~K} / \mathrm{s}$ to different holding temperatures for isothermal $\gamma \rightarrow \alpha$ transformation. The kinetics of the isothermal transformation can be derived by lever rule according to the dilation curves. It is worth mentioning that the massive $\gamma \rightarrow \alpha$ transformation during cooling after the isothermal holding cannot be avoided in present experiments, even if the largest cooling rate $(>200 \mathrm{~K} / \mathrm{s})$ of the dilatometer has been tried.

\section{Model Description}

In this study, a phase-field method, which takes interfacial segregation into account, is proposed to simulate the $\gamma \rightarrow \alpha$ transformation in $\mathrm{Fe}-2 \mathrm{Mn}$ and $\mathrm{Fe}-2 \mathrm{Mn}-0.5 \mathrm{Mo}$ alloys. The total Gibbs energy of a ternary system is given by

$G=\int_{\Omega}\left[\frac{1}{V_{\mathrm{m}}} G_{\mathrm{m}}\left(\phi, x_{i}\right)+\frac{1}{2} \varepsilon^{2}|\nabla \phi|^{2}\right] \cdot \mathrm{d} V$,

where $G_{\mathrm{m}}$ is the molar Gibbs energy. $x_{i}$ is the mole fraction of component $i(i=1,2,3) . V_{\mathrm{m}}$ is the molar volume of the system. $\varepsilon^{2}$ is the gradient coefficient. $\phi$ is the phase-field variable with value 0 in $\alpha$ phase and value 1 in $\gamma$ phase. Therefore, the value of $\phi$ at the interface region between the two phases continuously changes from 0 to 1 , and the molar Gibbs energy can be written as

$G_{\mathrm{m}}\left(\phi, x_{i}\right)=G_{\mathrm{m}}^{\alpha}[1-p(\phi)]+G_{\mathrm{m}}^{\gamma} p(\phi)+W\left(x_{i}\right) g(\phi)$.

Here, a monotonic function $p(\phi)=\phi^{3}\left(10-15 \phi+6 \phi^{2}\right)$ that has $p(0)=0$ and $p(1)=1$ reproduces the molar

Table 1 Chemical compositions and $T_{0}$ for the two alloys

\begin{tabular}{lllll}
\hline Alloy & \multicolumn{3}{l}{ Composition (wt\%) } & $T_{0}\left({ }^{\circ} \mathrm{C}\right)$ \\
\cline { 2 - 4 } & $\mathrm{Mn}$ & $\mathrm{Mo}$ & $\mathrm{Fe}$ & \\
\hline Fe-2Mn & 2.08 & - & Bal. & 802 \\
Fe-2Mn-0.5Mo & 2.11 & 0.49 & Bal. & 809 \\
\hline
\end{tabular}

Gibbs energy of each phase. A double well potential $g(\phi)=\phi^{2}(1-\phi)^{2}$ with its height $W\left(x_{i}\right)$ is adopted within the interface region. Taking $\mathrm{Fe}-\mathrm{Mn}-\mathrm{Mo}$ system as an example, the molar Gibbs energy of each phase can be described by regular solution model as

$G_{\mathrm{m}}^{\varphi}=\sum_{i} x_{i}^{\varphi 0} G_{i}^{\varphi}+R T \sum_{i} x_{i}^{\varphi} \ln x_{i}^{\varphi}+\sum_{i} \sum_{j} \Omega_{i j}^{\varphi} x_{i}^{\varphi} x_{j}^{\varphi}+\Omega_{123}^{\varphi} x_{1}^{\varphi} x_{2}^{\varphi} x_{3}^{\varphi}$,

where $\varphi=\alpha, \gamma$ represents different phases. ${ }^{0} G_{i}^{\varphi}$ is the molar Gibbs energy of pure $i$ in standard state in $\varphi$ phase. $x_{i}^{\varphi}$ is the mole fraction of $i$ in $\varphi$ phase. $R$ is the universal gas constant, and $T$ is the absolute temperature. The term $\Omega_{123}^{\varphi} x_{1}^{\varphi} x_{2}^{\varphi} x_{3}^{\varphi}$ represents the mixing molar Gibbs energy of $\varphi$ phase. In order to take interfacial segregation into account, $W\left(x_{i}\right)$ should decrease if the solute concentration at interface increases. According to the assumption for GB segregation [18] and interface segregation in binary alloys [10], a linear concentration dependency is introduced in $W$ by

$W\left(x_{i}\right)=w\left(1-\sum m_{i} \cdot x_{i}\right)$,

where both $w$ and $m_{i}$ are positive constants. The solute will tend to segregate to the interface to lower the interfacial energy. The coefficient $m_{i}$ describes the interaction between component $i$ and interface, and determines the binding energy $E_{0}$ which can be obtained from first-principle calculations or the commonly used values in the literature.

The temporal evolution equation of non-conserved phase-field variable $\phi$ is governed by Allen-Cahn equation [19], combining with Eq. (1), we have

$\frac{\partial \phi}{\partial t}=-M_{\phi} \frac{\delta G}{\delta \phi}=M_{\phi}\left(\varepsilon^{2} \nabla^{2} \phi-\frac{1}{V_{\mathrm{m}}} \frac{\partial G_{\mathrm{m}}}{\partial \phi}\right)$.

Here, $M_{\phi}=\left(\sigma / \varepsilon^{2}\right) M_{\text {int }}$ is a parameter related to the intrinsic interface mobility $M_{\text {int }} . \sigma$ is the interfacial energy.

The temporal evolution equation of conserved concentration $x_{i}$ can be obtained according to mass balance equation. Regarding Eq. (1), we have

$\frac{1}{V_{\mathrm{m}}} \frac{\partial x_{i}}{\partial t}=-\nabla \cdot J_{i}$

where $J_{i}$ is the flux of component $i$. Then, $J_{i}$ can be obtained from Onsager equation:

$J_{i}=-\sum_{j=1}^{N-1} L_{i j}^{\prime \prime} \nabla\left(\frac{\delta G}{\delta x_{i}}\right)$,

$L_{i j}^{\prime \prime}$ is a matrix related to diffusion mobility $M_{i}$, which can be expressed by [20,21]: 
$L_{i j}^{\prime \prime}=\sum_{k=1}^{N} \sum_{r=1}^{N}\left(\delta_{i k}-x_{i}\right)\left(\delta_{j r}-x_{j}\right) L_{k r}$

Here, if $i=j$, then $L_{i i}=x_{i} M_{i}$; if $i \neq j$, then $L_{i j}=0 . M_{i}$ at interface is assumed to be:

$M_{i}=(1-p(\phi)) M_{i}^{\alpha}+p(\phi) M_{i}^{\gamma}$,

where $M_{i}$ can be obtained with diffusivity $D_{i}$ by Einstein's relation $M_{i}=D_{i} / R T$.

In the following, a two-phase system with evenly distributed solute is considered, and the interface is assumed to be $1 \mathrm{~nm}$ thick. All the thermodynamic data used in the simulations are obtained from Thermo-Calc with TCFE7 and MOB2 database. The governing equations, i.e., Eqs. (5) and (6), are solved in their dimensionless forms using Fourier spectral method [22]. The length is normalized as $\tilde{z}=z / h$, where $h=0.2 \mathrm{~nm}$ is the grid size. The time is normalized as $\tilde{t}=t \cdot D_{0} / h^{2}$, where $D_{0}=M_{i}^{\alpha} R T$ is chosen as a reference diffusivity. The reference energy is obtained as $R T$. The values of simulation parameters are given as $V_{\mathrm{m}}=7 \times 10^{-6} \mathrm{~m}^{3} / \mathrm{mol}, \varepsilon^{2}=4 \times 10^{-10} \mathrm{~J} / \mathrm{m}, \sigma=0.3 \mathrm{~J} / \mathrm{m}^{2}$, $w=3 \times 10^{4} \mathrm{~J} / \mathrm{mol}$. The $\alpha / \gamma$ interface mobility is expressed as $M_{\text {int }}=2.7 \times 10^{-6} \exp (-145000 / R T) \mathrm{m}^{4} /(\mathrm{Js})$ [7]. The binding energies of $\mathrm{Mn}$ and Mo are set to be 8 and $22 \mathrm{~kJ} / \mathrm{mol}$, respectively, which are comparable with those reported in the literature for Mn [2, 23] and Mo [24, 25].

\section{Results and Discussion}

The measured kinetics of isothermal $\gamma \rightarrow \alpha$ transformation in the $\mathrm{Fe}-2 \mathrm{Mn}$ and $\mathrm{Fe}-2 \mathrm{Mn}-0.5 \mathrm{Mo}$ alloys at different temperatures is shown in Fig. 1. It can be seen that there is almost no transformation within $200 \mathrm{~s}$ at $760{ }^{\circ} \mathrm{C}$ for both alloys. However, when the temperature is down to $700{ }^{\circ} \mathrm{C}$, the transformation almost finishes within $30 \mathrm{~s}$ for the $\mathrm{Fe}-2 \mathrm{Mn}$ alloys and transforms about $80 \%$ in $60 \mathrm{~s}$ for the $\mathrm{Fe}-2 \mathrm{Mn}-0.5 \mathrm{Mo}$ alloys.

Using Eq. (10), the $\alpha / \gamma$ interface velocity can be estimated from the transformation curves (linear stage) in Fig. 1 according to Refs. $[6,7,17]$ :

$v_{\alpha / \gamma}=\frac{\mathrm{d} f_{\alpha} / \mathrm{d} t}{3\left(N^{*} \cdot g\right)^{1 / 3}\left(1-f_{\alpha}^{2}\right) \operatorname{arctanh}^{2 / 3}\left(f_{\alpha}\right)}$.

Here, $v_{\alpha / \gamma}$ is the $\alpha / \gamma$ interface velocity; $f_{\alpha}$ is the fraction of ferrite; $N^{*}$ is the density of nuclei per volume (it can be estimated as $d^{-3}$, and $d$ is the average grain size of ferrite); and $g$ is the geometrical factor ( $g=1$ for cubic growth and $g=4 \pi / 3$ for spherical growth).

Then, in Fig. 2a, the experimental interface velocity is compared with the PFM prediction as a function of temperature. The PFM predicts three kinetic modes for
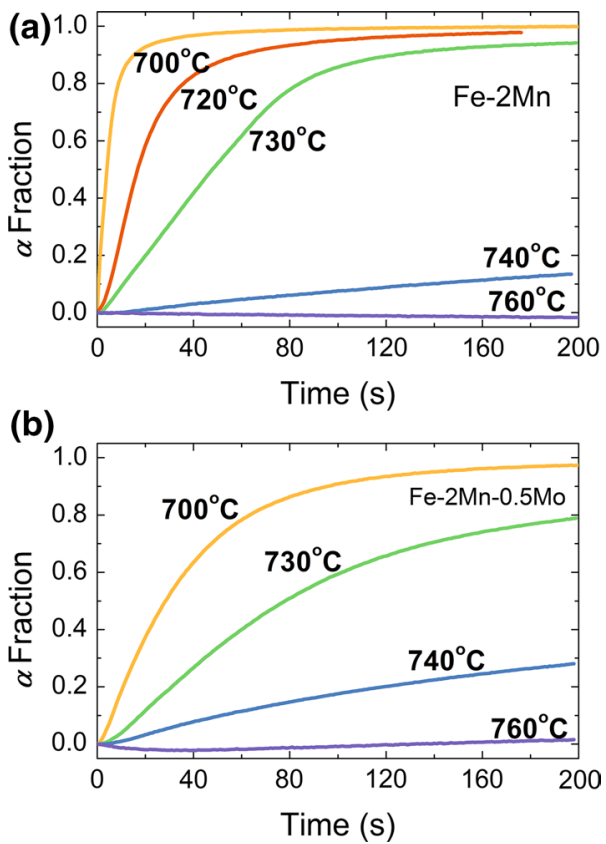

Fig. 1 Ferrite $(\alpha)$ fraction as a function of time during isothermal holding at various temperatures in a Fe-2Mn, b $\mathrm{Fe}-2 \mathrm{Mn}-0.5 \mathrm{Mo}$ alloys

the $\gamma \rightarrow \alpha$ transformation at different temperatures, which generally agrees with the experimental results. The $\mathrm{Mn}$ profiles at three typical temperatures are shown to demonstrate the kinetic modes. In Fig. 2b, there is evident Mn partitioning from $\alpha$ to $\gamma$ when the temperature is relatively higher (e.g., $780^{\circ} \mathrm{C}$ ), which is a partitioning transformation (denoted as mode I). The kinetics of interface migration in mode I is controlled by Mn diffusion in $\gamma$. Besides, in Fig. 2d, Mn hardly diffuses between the two phases nor segregates to the interface at relatively lower temperature (e.g., $700{ }^{\circ} \mathrm{C}$ ) during the transformation, which is a partitionless massive transformation (denoted as mode III). Transformation kinetics in mode III is interface-controlled. The partitioning and partitionless transformation modes have already been predicted and experimentally detected during the $\gamma \rightarrow \alpha$ transformation upon continuous cooling $[3,7,26]$.

At an intermediate temperature (e.g., $740{ }^{\circ} \mathrm{C}$ ), significant interfacial segregation of $\mathrm{Mn}$ is predicted as shown in Fig. 2c. The Mn spike is about $1 \mathrm{~nm}$ thick, which is inside the interface, and there is no obvious Mn partitioning (denoted as mode II). Correspondingly, interface velocity in mode II at $740{ }^{\circ} \mathrm{C}$ is somewhat between that in mode I and mode III. To the authors' best knowledge, a new type of transformation mode between sluggish partitioning transformation and fast massive transformation is found here. Different from mode I, there is only interfacial segregation of Mn but no Mn partitioning between $\gamma$ and $\alpha$ in 

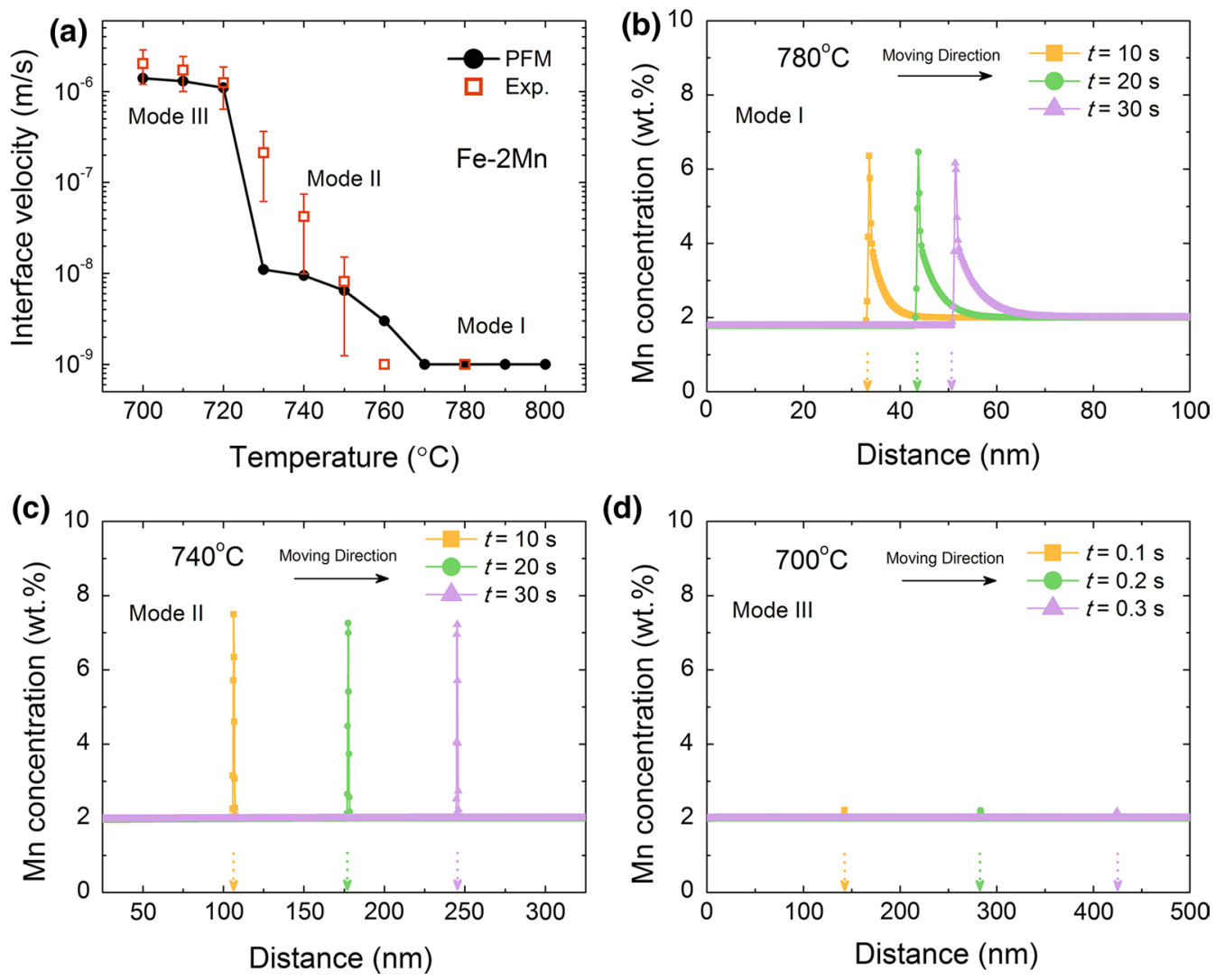

Fig. 2 Austenite $(\gamma)$-to-ferrite $(\alpha)$ transformation in an Fe-2Mn alloy, a the experimental $\alpha / \gamma$ interface velocity compared with PFM predictions at different temperatures, and the predicted Mn profiles at b $780{ }^{\circ} \mathrm{C}, \mathbf{c} 740{ }^{\circ} \mathrm{C}, \mathbf{d} 700{ }^{\circ} \mathrm{C}$

mode II. The kinetics of interface migration is controlled by interfacial diffusion of $\mathrm{Mn}$ when the transformation is in mode II.

Similar PFM simulations have also been carried out for the Fe-2Mn-0.5Mo alloys. As shown in Fig. 3a, compared with the experimental results, the kinetic transition from 780 to $700{ }^{\circ} \mathrm{C}$ has also been predicted by PFM simulations. The Mn and Mo profiles at different typical temperatures are shown in Fig. 3. In Fig. 3b, there is evident Mn partitioning from $\alpha$ to $\gamma$ phase as well as interfacial segregation of both Mo and Mn when the temperature is relatively higher (e.g., $780{ }^{\circ} \mathrm{C}$, mode I), which is a partitioning transformation mode (denoted as mode I). In Fig. 3d, neither Mn nor Mo diffusion occurs at relatively lower temperature (e.g., $700{ }^{\circ} \mathrm{C}$ ), which is a partitionless massive transformation mode (denoted as mode III). At an intermediate temperature (e.g., $740{ }^{\circ} \mathrm{C}$, mode II), significant segregation of both $\mathrm{Mn}$ and Mo is predicted to occur, which is within the interfacial region, while there is no solute partitioning between $\gamma$ and $\alpha$, as indicated in Fig. 3c. Therefore, an intermediate type of $\gamma \rightarrow \alpha$ transformation kinetics between sluggish partitioning transformation and fast partitionless transformation is also experimentally found in $\mathrm{Fe}-2 \mathrm{Mn}-0.5 \mathrm{Mo}$ alloys. Based on PFM predictions, the distribution of $\mathrm{Mn}$ at different temperatures in $\mathrm{Fe}-2 \mathrm{Mn}-0.5 \mathrm{Mo}$ alloys is similar to that in $\mathrm{Fe}-2 \mathrm{Mn}$ alloys.

Mo is widely added to retard $\gamma \rightarrow \alpha$ transformation, and it has been reported in the literature that even $0.5 \mathrm{wt} \% \mathrm{Mo}$ addition has great influence on the kinetics of $\gamma \rightarrow \alpha$ transformation in carbon-contained steels [13, 27]. However, in our study, comparing the experimental results of two interstitialfree steels in Fig. 4, it is interestingly found that the addition of $0.5 \mathrm{wt} \%$ Mo has a marginal effect on the transformation kinetics. In mode I (e.g., $780^{\circ} \mathrm{C}$ ), i.e., the partitioning mode, the kinetics of interface migration is controlled by the diffusion of Mn and Mo in $\gamma$, which is extremely sluggish. In mode II (e.g., $740{ }^{\circ} \mathrm{C}$ ), the kinetics of interface migration is controlled by the interfacial diffusion of Mn and Mo. The difference between interfacial diffusion of $\mathrm{Mn}$ and Mo could be negligible, which could explain why interface velocities for $\mathrm{Fe}-2 \mathrm{Mn}$ and $\mathrm{Fe}-2 \mathrm{Mn}-0.5 \mathrm{Mo}$ alloys in mode II are similar. In mode III (e.g., $700{ }^{\circ} \mathrm{C}$ ), i.e., the partitionless mode, the kinetics of interface migration is controlled by the intrinsic interface mobility and chemical driving force for the $\gamma \rightarrow \alpha$ transformation. Mo addition will not affect the intrinsic $\alpha / \gamma$ interface mobility. Besides, the chemical potential of Mo in $\alpha$ is quite comparable to that in $\gamma$, and thus Mo has a small effect on chemical driving force $[28,29]\left(T_{0}\right.$ changes only $7^{\circ} \mathrm{C}$ with 

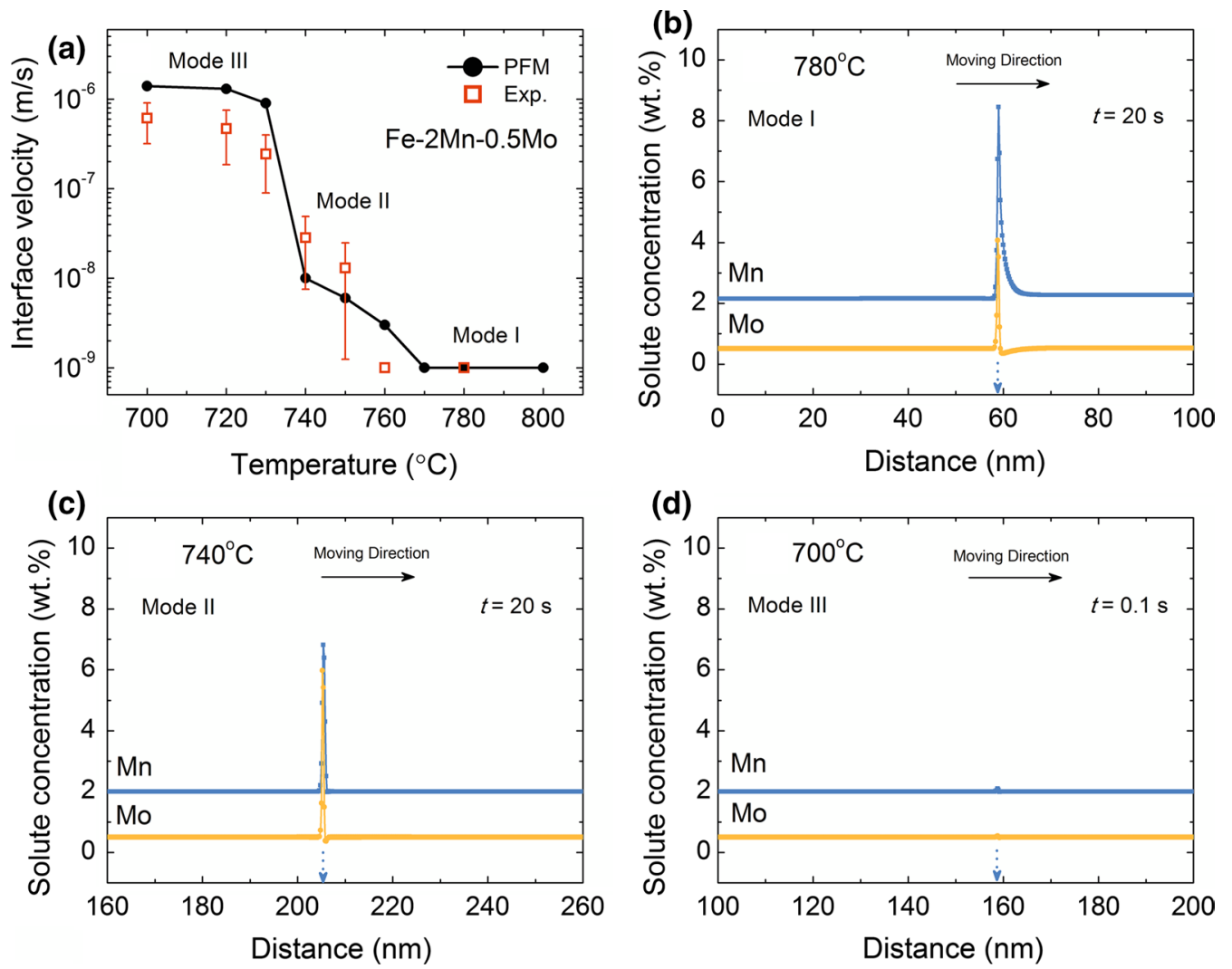

Fig. 3 Austenite $(\gamma)$-to-ferrite $(\alpha)$ transformation in an Fe-2Mn-0.5Mo alloy, a the experimental $\alpha / \gamma$ interface velocity compared with PFM predictions at different temperatures, and the predicted solute profiles at different time at $\mathbf{b} 780{ }^{\circ} \mathrm{C}, \mathbf{c} 740{ }^{\circ} \mathrm{C}, \mathbf{d} 700{ }^{\circ} \mathrm{C}$ (the arrows on the axis indicate the positions of interface)

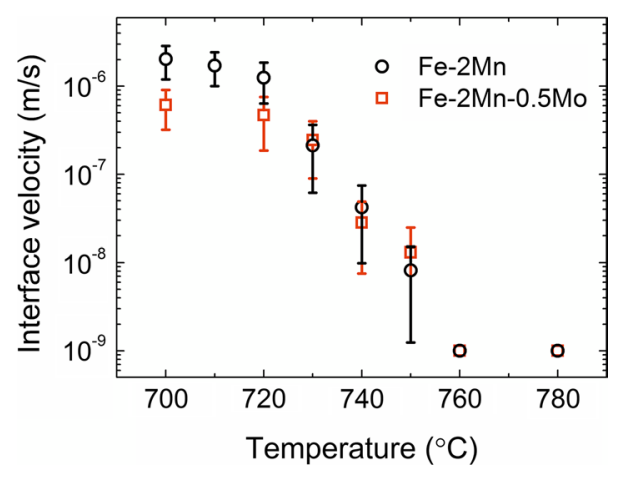

Fig. 4 Experimental $\alpha / \gamma$ interface velocity during $\gamma \rightarrow \alpha$ transformation in an $\mathrm{Fe}-2 \mathrm{Mn}$ alloy and an $\mathrm{Fe}-2 \mathrm{Mn}-0.5 \mathrm{Mo}$ alloy

$0.5 \mathrm{wt} \%$ Mo addition according to Table 1). Therefore, Mo addition hardly changes the transformation kinetic modes of Fe-2Mn alloys.

\section{Conclusions}

In summary, the isothermal $\gamma \rightarrow \alpha$ transformation at various temperatures in the $\mathrm{Fe}-2 \mathrm{Mn}$ and $\mathrm{Fe}-2 \mathrm{Mn}-0.5 \mathrm{Mo}$ alloys has been studied. Mo addition is experimentally found to have a marginal effect on the transformation kinetics. It is also found that the transition from partitioning to partitionless transformation is not sharp. There can be a new kinetic mode, e.g., interfacial diffusion-controlled mode, in which the interface migrates at an intermediate velocity between sluggish partitioning transformation and fast partitionless transformation. The complex transition of kinetic modes could be well predicted by a phase-field model with considering interfacial segregation.

Acknowledgements Z.-G. Yang acknowledges the financial support from the National Natural Science Foundation of China (Grant Nos. U174252, 51771100). H. Chen acknowledges the financial support from National Natural Science Foundation of China (Grant Nos. U1808208, U1860109, 51922054), the Beijing Natural Science Foundation (No. 2182024) and the National Key R\&D program of China (No. 2016YFB0300104). C. Zhang acknowledges the financial support from the National Natural Science Foundation of China (Grant No. 2015CB654802), the National Magnetic Confinement Fusion Energy Research Project of China (Grant No. 2015GB118001) and the Fund 
of Key Laboratory of Advanced Materials of Ministry of Education (Grant No. 2017AML09).

\section{References}

[1] J. Sietsma, S. Van Der Zwaag, Acta Mater. 52, 4143 (2004)

[2] H. Chen, S. van der Zwaag, Acta Mater. 72, 1 (2014)

[3] Y. Liu, F. Sommer, E.J. Mittemeijer, Acta Mater. 54, 3383 (2006)

[4] G. Purdy, J. Ågren, A. Borgenstam, Y. Bréchet, M. Enomoto, T. Furuhara, E. Gamsjager, M. Gouné, M. Hillert, C. Hutchinson, M. Militzer, H. Zurob, Metall. Mater. Trans. A 42, 3703 (2011)

[5] M. Gouné, F. Danoix, J. Ågren, Y. Bréchet, C.R. Hutchinson, M. Militzer, G. Purdy, S. Van Der Zwaag, H. Zurob, Mater. Sci. Eng. R Rep. 92, 1 (2015)

[6] Y. Liu, F. Sommer, E. Mittemeijer, Acta Mater. 52, 2549 (2004)

[7] J. Zhu, H. Luo, Z. Yang, C. Zhang, S. van der Zwaag, H. Chen, Acta Mater. 133, 258 (2017)

[8] G.P. Krielaart, S.V.D. Zwaag, Mater. Sci. Technol. 14, 10 (1998)

[9] A. Borgenstam, M. Hillert, Acta Mater. 48, 2765 (2000)

[10] C.Y. Zhang, H. Chen, J.N. Zhu, W.B. Liu, G. Liu, C. Zhang, Z.G. Yang, Scr. Mater. 162, 44 (2019)

[11] H.S. Zurob, C.R. Hutchinson, A. Béché, G.R. Purdy, Y.J.M. Bréchet, Acta Mater. 56, 2203 (2008)

[12] H. Chen, Z.G. Yang, C. Zhang, K. Zhu, S. Van Der Zwaag, Acta Mater. 104, 62 (2016)

[13] G. Miyamoto, K. Yokoyama, T. Furuhara, Acta Mater. 177, 187 (2019)
[14] Y. Xia, G. Miyamoto, Z.G. Yang, C. Zhang, T. Furuhara, Metall. Mater. Trans. A 46, 2347 (2015)

[15] W.W. Sun, H.S. Zurob, C.R. Hutchinson, Acta Mater. 139, 62 (2017)

[16] J. Hamada, M. Enomoto, T. Fujishiro, T. Akatsuka, Metall. Mater. Trans. A 45, 3781 (2014)

[17] A.T.W. Kempen, F. Sommer, E.J. Mittemeijer, Acta Mater. 50, $3545(2002)$

[18] K. Grönhagen, J. Ågren, Acta Mater. 55, 955 (2007)

[19] S.M. Allen, J.W. Cahn, Acta Metall. 27, 1085 (1979)

[20] J.O. Andersson, J. Ågren, J. Appl. Phys. 72, 1350 (1992)

[21] A. Malik, J. Odqvist, L. Höglund, S. Hertzman, J. Ågren, Metall. Mater. Trans. A 48, 4914 (2017)

[22] L.Q. Chen, J. Shen, Comput. Phys. Commun. 108, 147 (1998)

[23] M. Enomoto, C.L. White, H.I. Aaronson, Metall. Trans. A 19, 1807 (1988)

[24] H. Jin, I. Elfimov, M. Militzer, J. Appl. Phys. 115, 093506 (2014)

[25] H.P. Van Landeghem, B. Langelier, B. Gault, D. Panahi, A. Korinek, G.R. Purdy, H.S. Zurob, Acta Mater. 124, 536 (2017)

[26] I. Loginova, J. Odqvist, G. Amberg, J. Ågren, Acta Mater. 51, $1327(2003)$

[27] Y. Xia, G. Miyamoto, Z.G. Yang, C. Zhang, T. Furuhara, Acta Mater. 91, 10 (2015)

[28] H. Chen, S. Van Der Zwaag, Metall. Mater. Trans. A 45, 3429 (2014)

[29] C.Y. Zhang, H. Chen, K. Zhu, C. Zhang, Z.G. Yang, Metall. Mater. Trans. A 47, 5670 (2016) 